\title{
THE INTEGRATED MAGNETIC FIELD OF THE SUN SEEN BY THE GOLF EXPERIMENT
}

\author{
R. A. GARCÍA ${ }^{1}$, T. ROCA CORTÉS ${ }^{2}$ AND THE GOLF TEAM ${ }^{\dagger}$ \\ ${ }^{1}$ SAp, DAPNIA/DSM, CE Saclay. 91191 France \\ ${ }^{2}$ Instituto de Astrofisica de Canarias, E-38205, Tenerife, Spain \\ 'GOLF Team: P. Boumier, J. Charra, A.H. Gabriel, G. Grec, J.M. Robillot, \\ S. Turck-Chièze, R.K. Ulrich
}

\begin{abstract}
The secondary objective of the GOLF experiment on-board the $\mathrm{SOHO}$ space mission is to measure the line-of-sight component of the disk averaged magnetic field of the Sun. GOLF is an improved disk-integrated sunlight resonant scattering spectrophotometer. Using an extra fixed quarter wave plate placed at the entrance of the instrument, enables a selection of the circularly polarized solar light and therefore, the disk averaged solar line-of-sight component of the magnetic field can be obtained. Unfortunately, due to occasional malfunction of the rotating mechanisms, only a series of 26 continuous dar's are available. Here, the analysis of this series is presented including the value of the areraged magnetic field.
\end{abstract}

\section{Method}

The method of obtaining the magnetic field is based on the measure of the $I \pm V$ Stokes parameters (a full description is in García (1996)). If we assume that the solar magnetic field is weak throughout the atmosphere where the sodium doublet is formed, we can use the so-called weak field approximation. In this case, the displacement due to the solar magnetic field, $\delta \lambda_{B \odot}$ is much smaller than the Doppler width of the original line profile (for a general description see e.g. Stix 1989). In this approximation, we can consider the magnetic field as a small perturbation and make a Taylor expansion of the Stokes parameters in $\delta \lambda_{B \odot}$, up to first order. Adding and substracting the non zero Stokes parameters, i.e. $I$ and $V$ :

$$
I \pm V=I\left(\lambda_{o}\right) \pm \delta \lambda_{B \odot} \cdot\left(\frac{d I}{d \lambda}\right)_{\lambda_{0}}
$$

Each GOLF measurement is a combination of this two parameters: $I_{b}^{-}, I_{b}^{+}, I_{r}^{-}, I_{r}^{+}$ which correspond to $I-V$ and $I_{b}^{\prime}, I_{b}^{\prime}, I_{r}^{\prime}, I_{r}^{\prime}+$ to $I+V$; where $I^{\prime}$ denotes the 
$\sigma^{-}$polarized component of the solar light and $\lambda_{0}$ denotes the longitudinal Zeeman wavelength.

Substracting equations 1, and expressing each magnitude in terms of GOLF observables we can obtain an expression that links the longitudinal component of the global magnetic field of the Sun with the GOLF measurements:

$$
B_{\odot}=\frac{\delta B}{2} \cdot\left[\frac{\left(I_{b}^{\prime}+-I_{b}^{+}+I_{b}^{\prime}-I_{b}^{-}\right)}{\left(I_{b}^{\prime+}-I_{b}^{\prime}+I_{b}^{+}-I_{b}^{-}\right)}-\frac{\left(I_{r}^{\prime}--I_{r}^{-}+I_{r}^{\prime+}-I_{r}^{+}\right)}{\left(I_{r}^{\prime+}-I_{r}^{\prime-}+I_{r}^{+}-I_{r}^{-}\right)}\right]
$$

The validity of this method has been tested using a numerical simulation of the GOLF experiment (Carcía, Roca Cortés and Régulo 1997). The simulated magnetic field was recovered with a precision better than 2 parts in 1000 .

\section{GOLF magnetic field observations}

In Fig. 1, the resultant solar magnetic field is plotted as a function of time. A fitting of the form $A \cdot \sin (t / P+\phi)+c$ has been calculated giving a main periodicity of $\simeq 13.8$ days with an amplitude $A \simeq 0.281$ and an offset of $c \simeq 0.149 G$.
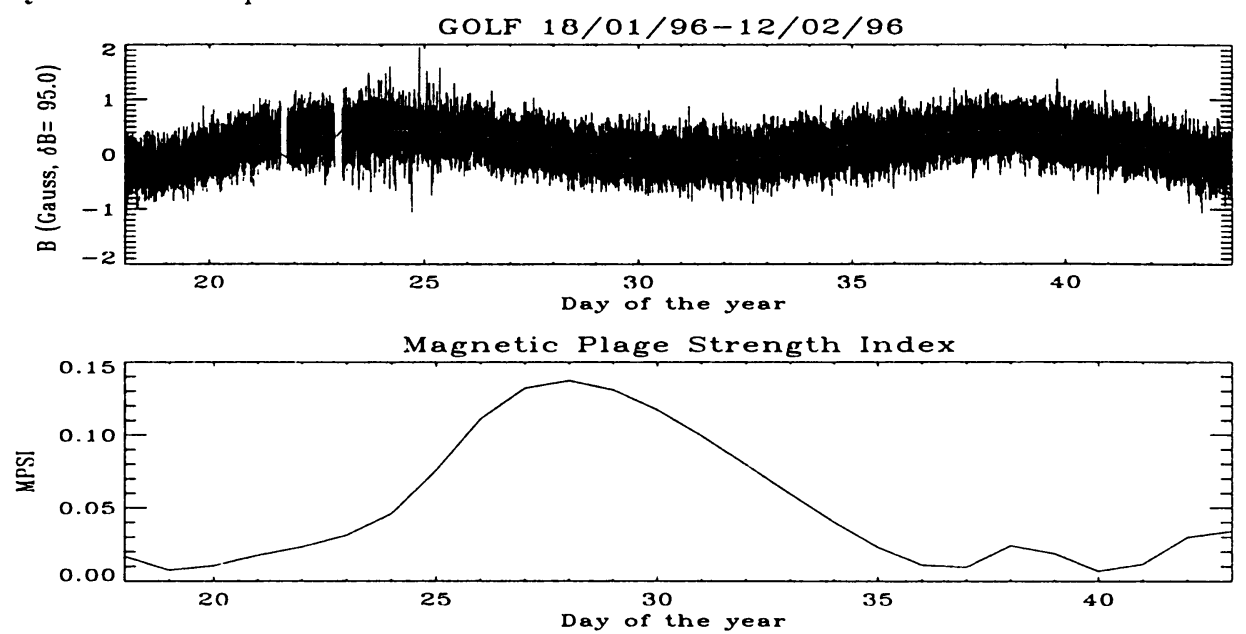

Figure 1. Up: Longitudinal global magnetic field of the Sun computed using equation 2. The gray line shows the best sinusoidal fit. Down: MPSI magnetic index for the same period of time.

This periodicity is related to solar rotation. During this period of time the Mount Wilson MPSI magnetic index (see the lower graph) shows the passage of an active region over the solar disk. The small phase shift ( $\sim 3$ days $)$ is well explained by the different weighting functions of the two instruments.

\section{References}

-García R.A., 1996, PhD Thesis, Univ. La Laguna, Tenerife, Spain -García, R.A., Roca Cortés, T. and Régulo C. 1997, Astron. Astrophys. Supp. in Press -Stix, M. 1989, The Sun. An Introduction, Springer-Verlag 